\title{
INSTRUMENTOS MATEMÁTICOS DO TRATADO TECTONICON: uma possibilidade de trabalho em sala de aula
}

\author{
Ana Rebeca Miranda Castillo* \\ Ângela Maria dos Santos **
}

\section{Resumo}

Neste trabalho discorremos sobre os conhecimentos matemáticos incorporados e mobilizados no uso do esquadro geométrico e bastão de carpinteiro, instrumentos de medida abordados no tratado A Boke Named Tectonicon, escrito e publicado por Leonard Digges (1520-1559). Temos como objetivos: a partir dos procedimentos descritos pelo autor, identificar e analisar os conhecimentos matemáticos abordados no tratado e descrever uma possibilidade de uso da História da Matemática em sala de aula. Como abordagem metodológica, articulamos três dimensões de análise, a saber:historiográfica, contextual e epistemológica. Verificamos como eram utilizados os instrumentos para determinar o nivelamento de um objeto em relação ao observador, o cálculo da altura desse objeto e o cálculo de pequenas distâncias. Os resultados deste trabalho mostraram que esses instrumentos abordados em Tectonicon, incorporam conhecimentos e mostram a relação entre o saber e o fazer de uma época além de, apontar possibilidades de trabalho com conteúdo matemático da educação básica.

Palavras-chave: História da Matemática. Instrumentos Matemáticos. Régua de Carpinteiro. Esquadro de Carpinteiro. Leonard Digges.

\begin{abstract}
In this paper we discuss the mathematical knowledge incorporated and mobilized in the use of the geometric square and carpenter's rod, measuring instruments addressed in the treatise A Boke Named Tectonicon, written and published by Leonard Digges (1520-1559). We have as objectives: from the procedures described by the author, identify and analyze the mathematical knowledge addressed in the treatise and describe a possibility of using the History of Mathematics in the classroom. As a methodological approach we articulate three dimensions of analysis, namely: historiographic, contextual and epistemological. We verified how the instruments were used to determine the leveling of an object in relation to the observer, the calculation of that object's height and the calculation of short distances. The results of this work showed that these instru-

\footnotetext{
Pesquisadora Doutora Colaboradora (PDC) da Universidade Federal do ABC (UFABC) no Grupo de Pesquisa na Educação Matemática (GPTEMa). E-mail: ana.rebeca@ufabc.edu.br

** Doutora em Educação Matemática pela PUC SP. Professora do Instituto Federal de Ciência e Tecnologia (IFSP), campi Caraguatatuba. E-mail: profangelamariasantos@gmail.com
} 
Instrumentos matemáticos do Tratado TECTONICON: uma possibilidade de trabalho em sala de aula

ments approached in Tectonicon, incorporate knowledge and show the relationship between the knowledge and the doing of a time, besides pointing possibilities of work with mathematical content of basic education.

Keywords: History of Mathematics. Mathematical instruments. Carpenter's Ruler. Carpenter's Square. Leonard Digges.

\section{Introdução}

O papel dos instrumentos de medida durante o século XVI e início do XVII, provocou muitos debates. Para alguns estudiosos, o uso de um instrumento era um fator determinante para consolidar-se como um praticante das matemáticas ${ }^{1}$, já para outros não passavam de meios para "exibição de truques". Além disso, havia a questão de considerar se o uso de instrumentos levava a uma compreensão das matemáticas ou apenas fornecia respostas sem indicar de onde procediam (HIGTON, 2001).

Com a descoberta e conquista de novos territórios, o estabelecimento e demarcação de fronteiras se tornava urgente e com isso o cálculo de medidas de distâncias e de áreas era uma tarefa cada vez mais solicitada. Além disso, com a dissolução dos monastérios centenas de propriedades foram liberadas do poderio da igreja e as dispustas por propriedades passaram cada vez mais a ser decididas nos tribunais de Londres. O agrimensor era um trabalhador temporário mais especializado que trazia para a propriedade novos padrões de conhecimento e de organização (MCRAE 1993).

Os praticantes das matemáticas responderam a estas demandas elaborando técnicas, projetando novos instrumentos e publicando

\footnotetext{
${ }^{1}$ Os praticantes das matemáticas eram aqueles que não só praticavam as matemáticas, mas também construíam e manipulavam instrumentos matemáticos. Esta característica os distinguia de outros artesãos, pois, além de construir e manipular tais instrumentos, escreviam as instruções necessárias sobre como utilizá-los a fim de obter a medida satisfatoriamente. (Harkness, 2007; Richeson, 1966; Taylor, 1954).
} 
Instrumentos matemáticos do Tratado TECTONICON: uma possibilidade de trabalho em sala de aula

tratados. Entre eles, Leonard Digges ${ }^{2}$ (1520-1559) segundo Harkness (2007) foi um dos autores que direcionou seus trabalhos aos instrumentos matemáticos e aos conhecimentos geométricos necessários para sua fabricação e uso, sendo considerado pelos historiadores da ciência como pioneiro na produção de textos voltados para a prática geométrica do homem comum na Inglaterra do século XVI. No tratado, escrito por ele, intitulado A Boke Named Tectonicon ${ }^{3}$ (DIGGES, 1605), dedicado especialmente à agrimensura, foi publicado pela primeira vez em 1556 e apresenta uma série de procedimentos de medida que nos revela interessantes aspectos do saber-fazer matemático daquela época.

Tectonicon é dividido em duas seções precedidas por um texto dirigido ao leitor. A primeira seção é intitulada "Diversas coisas conduzidas na arte da medição", e é composta de vinte e um capítulos que fornecem basicamente as primeiras noções matemáticas requeridas para o ofício da agrimensura e também a descrição de como construir os instrumentos e na sequência como utilizá-los fornecendo exemplos e, por vezes, figuras que explicam o uso e como são feitos os cálculos para determinar a medida.

$\mathrm{Na}$ segunda e última seção, dividida em quatro capítulos, o autor explica como fabricar e utilizar um instrumento geométrico denominado por ele como báculo. Neste trabalho, optamos por descrever os conheci mentos matemáticos envolvidos no uso do esquadro geométrico - instrumento acoplado na parte de trás da régua de carpinteiro e no esquadro de carpinteiro. Ambos os instrumentos são abordados pelo autor a partir do capítulo 12 da primeira seção.

\footnotetext{
¿"Leonard Digges é uma figura bem conhecida pelos historiadores da ciência, porém pouco espaço lhe foi dedicado na história da matemática. Ele é considerado pelos historiadores da ciência pioneiro na produção de textos voltados para a prática geométrica do homem comum na Inglaterra do século XVI.A família de Leonard era do condado de Kent, Inglaterra, e se destacava por ser dona de várias propriedades porém. não era apenas um senhor de terras, mas sim um fidalgo, também poderíamos dizer que se tratava de um aristocrata rural" (CASTILLO, 2016, p.24).

${ }^{3}$ Conforme Van den Hoven (1996) e Amaral (2009), "Tectonicon” refere-se à tekton, termo que significa carpinteiro em grego antigo. Doravante, designaremos esta obra apenas por Tectonicon.
} 
Instrumentos matemáticos do Tratado TECTONICON: uma possibilidade de trabalho em sala de aula

\section{Régua de Carpinteiro e Esquadro Geométrico}

Uma demanda atendida pelo tratado Tectonicon é quanto à como saber o nivelamento de um terreno em relação a um determinado ponto de observação, já que nos novos territórios descobertos, como os países baixos, e nos pântanos ingleses ter água corrente era de grande importância econômica, e para isso agrimensores com seu conhecimento eram solicitados no auxílio para delinear cursos d'água, construir diques e comportas de contenção para resgatar terras para o plantio.

O instrumento apresentado no tratado que atenderia a essa finalidade é a régua de carpinteiro (Carpenters ruler), citada no capítulo doze de Tectonicon (figura 1). O autor não se detém em muitas explicações de como utilizá-la, apenas explica como produzi-la e como nomear suas escalas, tanto na parte da frente como na parte de trás da régua.
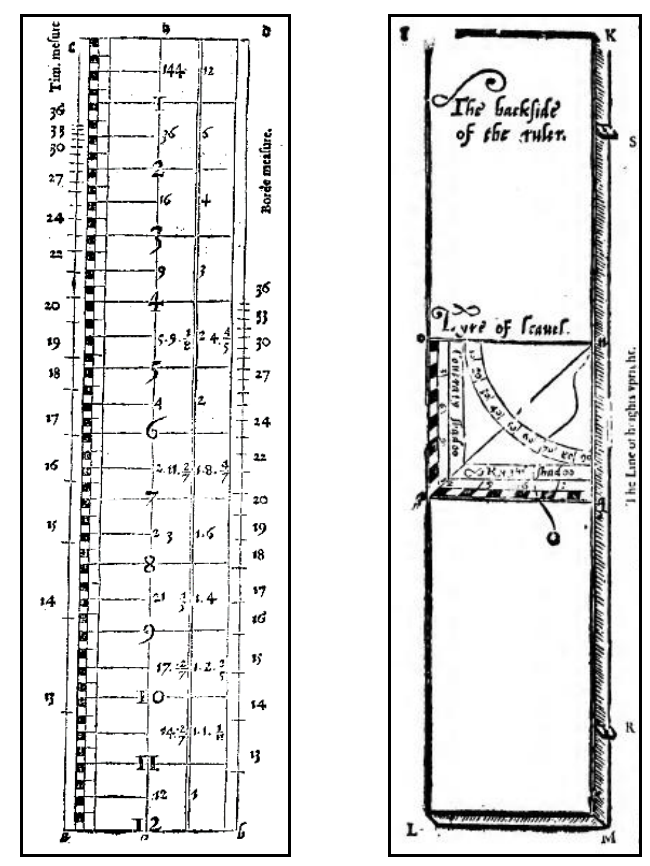

Figura 1 - Régua de carpinteiro, partes da frente e de trás Fonte: Digges (1605) 
Instrumentos matemáticos do Tratado TECTONICON: uma possibilidade de trabalho em sala de aula

Leonard inicialmente explica como dividir as escalas na parte da frente da régua, não nos deteremos nessas explicações já que nosso foco é o uso da parte de traz da régua onde o autor orienta que na metade da medida do comprimento da régua deverá ser colocado um esquadro geométrico. Nessas explicações o autor nomeia os vértices "n o p q", fornece as medidas das linhas "n o", "o p" denominada "sombra contrária", "p q" denominada "sombra reta" e "q n", nomeia as linhas "n p", "o n" e "q n" de "linha de altura", "linha de nivel" e "linha de altura vertical" respectivamente e orienta o traçado do quadrante circular dividido segundo o autor em 90 partes iguais que serão os graus. Finaliza explicando que poderão ser fixadas quando necessário duas vistas de madeira que ele denomina de "R" e "S". Na produção do esquadro geométrico, identificamos uma possibilidade de trabalho em sala de aula, se discutirmos o traçado geométrico necessário para sua produção (figura 2), as medidas angulares utilizadas e como elas influenciam na identificação do nivelamento de um terreno, já que é com essa parte de trás da régua que poderá ser verificado quando um terreno está nivelado o que é importante para por exemplo verificar se a água proveniente de uma fonte ou cabeceira pode ser conduzida a outro terreno e, além disso como por meio da ideia de proporcionalidade, podemos determinar a medida da altura de um objeto.

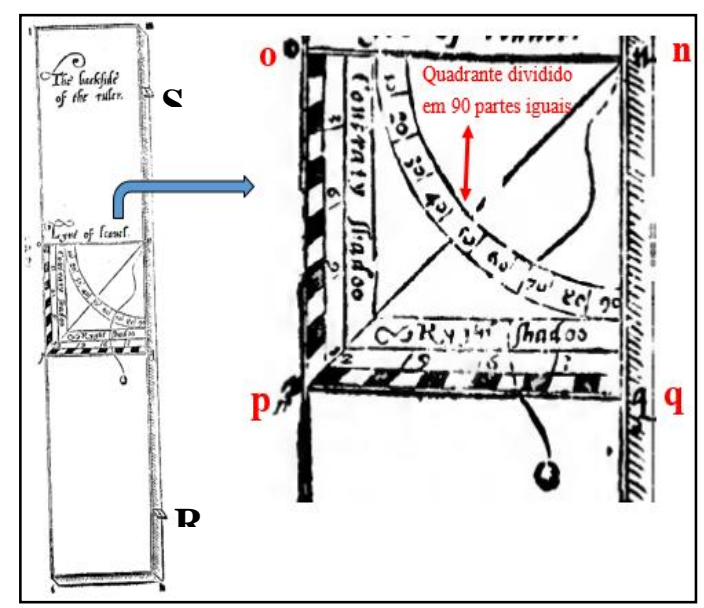

Figura 2 - Detalhes do esquadro geométrico

Fonte: Digges (1605, destaque nosso) 
Esse instrumento é utilizado somente como um indicador da posição do fio de prumo em relação a um objeto observado. No capítulo quinze de Tectonicon, são fornecidas as orientações de como utilizá-lo para verificar se um objeto está no mesmo nivel do observador ou não. Explica que ao olhar através das vistas "R e S", se o fio de prumo "cair" precisamente sobre a linha "n o", tudo o que é visto está nivelado com a posição do observador (figura 3a), se "cair" mais afastado do observador, o que estiver sendo observado estará abaixo do nível da posição do observador (figura 4b) e caso contrário, se "cair" menos afastado (figura 4c) estará nivelado acima da posição do observador.

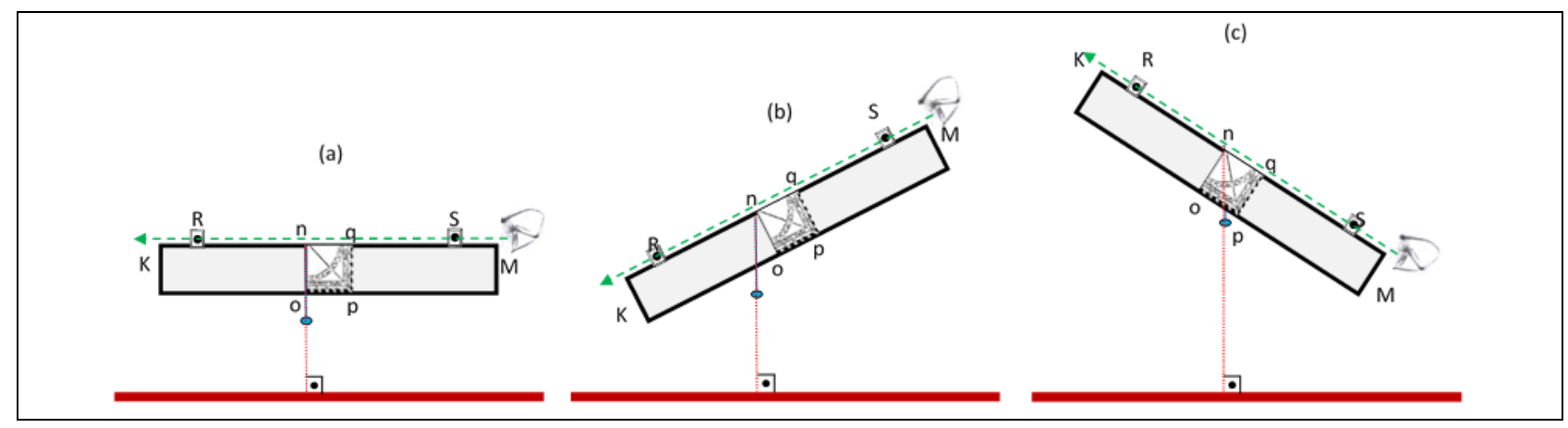

Figura 3 - Niveis com a régua de carpinteiro

Fonte: Produção nossa

No capitulo dezesseis, de Tectonicon, Leonard propõe utilizar a parte de trás da régua de carpinteiro para determinar a medida da altura de um objeto. Não fornece nenhuma justificativa matemática, mesmo porque não era necessária para a atividade do agrimensor, já que para sua prática ele apenas precisava saber como obter a medida da altura.

Uma possibilidade de trabalho em sala de aula com os conhecimentos envolvidos nas orientações fornecidas pelo autor é buscarmos a justificativa matemática que legitima o uso do instrumento para determinar a 
Instrumentos matemáticos do Tratado TECTONICON: uma possibilidade de trabalho em sala de aula

altura de um objeto. É possivel observar que o autor recorre à ideia de proporcionalidade, fundamentada em uma semelhança de triângulos formados pelo posicionamento do instrumento, especificamente em um ângulo de $45^{\circ}$, como podemos observar na figura 5 . Nela utilizamos a linguagem matemática moderna para explicar o que diz seu texto,

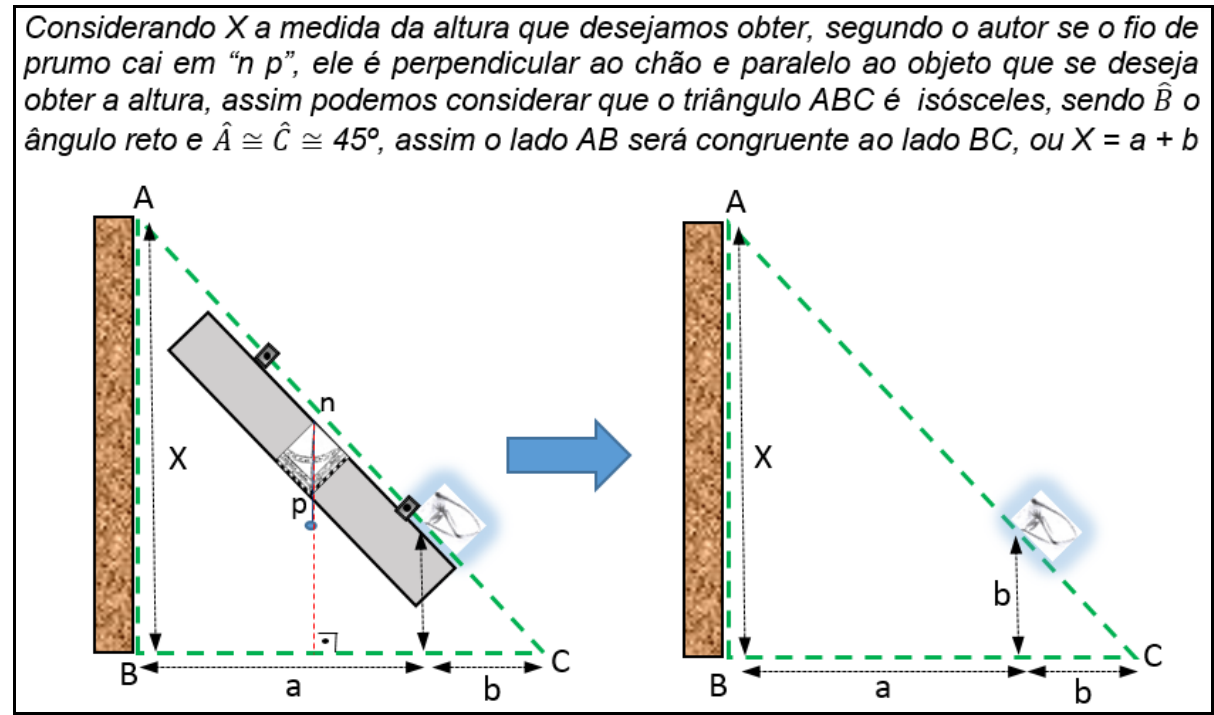

Figura 4 - Justificativa matemática medição de altura com a régua de carpinteiro Fonte: Elaboração das autoras

Se o fio de prumo não cair em "np", o triângulo formado não será isósceles e não será possível afirmar a medida da altura recorrendo à propriedade do triângulo isósceles. Em uma situação hipotética, seria possivel encontrar a medida da altura se o ângulo de inclinação fosse identificado e, a partir dele, o leitor recorresse a relações trigonométricas para a obtenção da medida da altura outra possibilidade de trabalho em sala de aula já que Leonard não fornece nenhuma indicação para isso. Podemos ainda trabalhar em sala de aula como encontrar a medida de alturas em que o fio de prumo se posicione nas escalas lineares "o p" e "p q", situações tratadas nos capítulos dezoito e dezenove, porém sem a ex- 
plicação de porque isso pode ser feito (uso novamente da ideia de proporcionalidade em triângulos semelhantes).

\section{Esquadro de Carpinteiro}

A ideia de proporcionalidade também é utilizada quando Leonard aborda o uso do esquadro de carpinteiro (figura 5) no capítulo vinte. Inicialmente o autor fornece as medidas do instrumento e neste momento identificamos uma nova possibilidade de trabalho em sala de aula quando aborda a unidade de medida em "minutos". O instrumento deverá ter um lado com dois pés e o outro com um pé. O lado maior, que Leonard denomina por "a b" deve ser dividido em 24 partes iguais e cada uma delas dividida em 10 minutos, a parte externa que chama de "c d" deve ser dividida em 12 partes iguais, cada uma destas dividida em 6 partes e estas por sua vez divididas em 10 minutos $^{4}$. Um fio de prumo "cai" de "e" para "f" paralelo a "c d" e "a b".

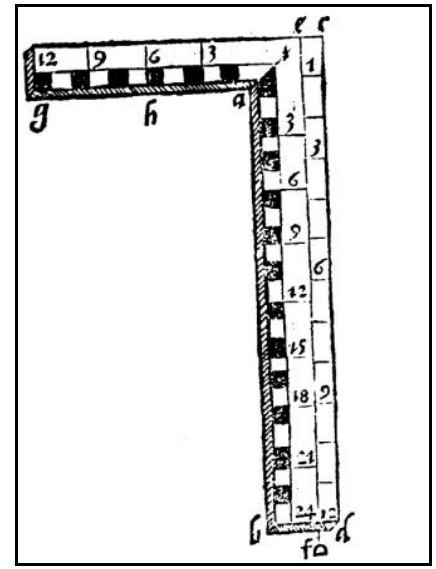

Figura 5 - Esquadro de carpinteiro Fonte: Digges (1605)

\footnotetext{
${ }^{4} \mathrm{O}$ autor faz referência à unidade de medida "minutos" como uma unidade de medida para uma escala linear, não fornece maiores explicações de como é estabelecida, e embora hoje sabidamente essa unidade de medida faça parte dos submúltiplos do grau, que é uma unidade de medida angular, não nos deteremos em aprofundar esta questão por não fazer parte dos objetivos de nosso trabalho.
} 
Instrumentos matemáticos do Tratado TECTONICON: uma possibilidade de trabalho em sala de aula

O capítulo seguinte orienta a como utilizar o esquadro para medir um comprimento em um terreno plano, explicando que será necessário um bastão (destaque na figura 6), no qual ficará apoiado o esquadro de carpinteiro, para que, assim, seja possivel avistar, através das vistas colocadas no esquadro, o ponto mais distante da longitude que se deseja medir, e na sequência fornece uma figura ilustrativa (figura 6).

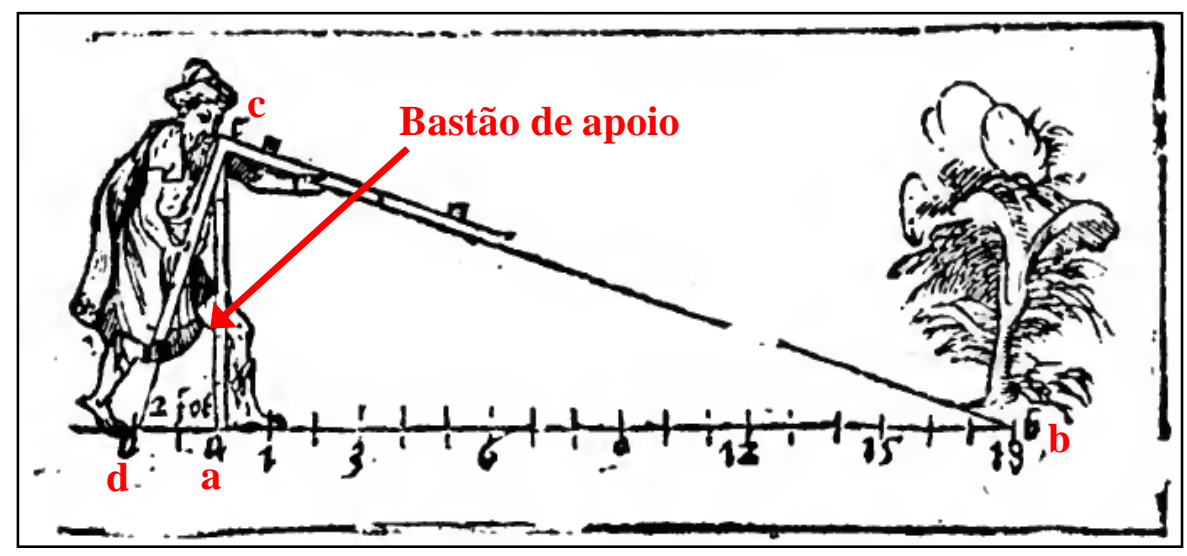

Figura 6 - Esquema explicativo para do esquadro de carpinteiro na medição de larguras

Fonte: Digges (1605, destaque nosso)

Leonard explica, em seu exemplo, que o bastão de apoio deverá ter 6 pés de comprimento e a partir disso o esquadro funcionará como um instrumento auxiliar para localizar o outro extremo da distância que se deseja medir. Como foi construído com um ângulo reto também possibilita, por meio dele, estabelecer as relações de proporcionalidade entre os valores das medidas " $a d$ " e " $a b$ " em relação à medida do bastão. A justificativa matemática para a obtenção da distância não é fornecida pelo autor, constituindo outra possibilidade de trabalho em sala de aula com o instrumento, como podemos verificar em linguagem moderna a possibilidade da figura 7 em que utilizamos os valores fornecidos no exemplo dado por Leonard. 


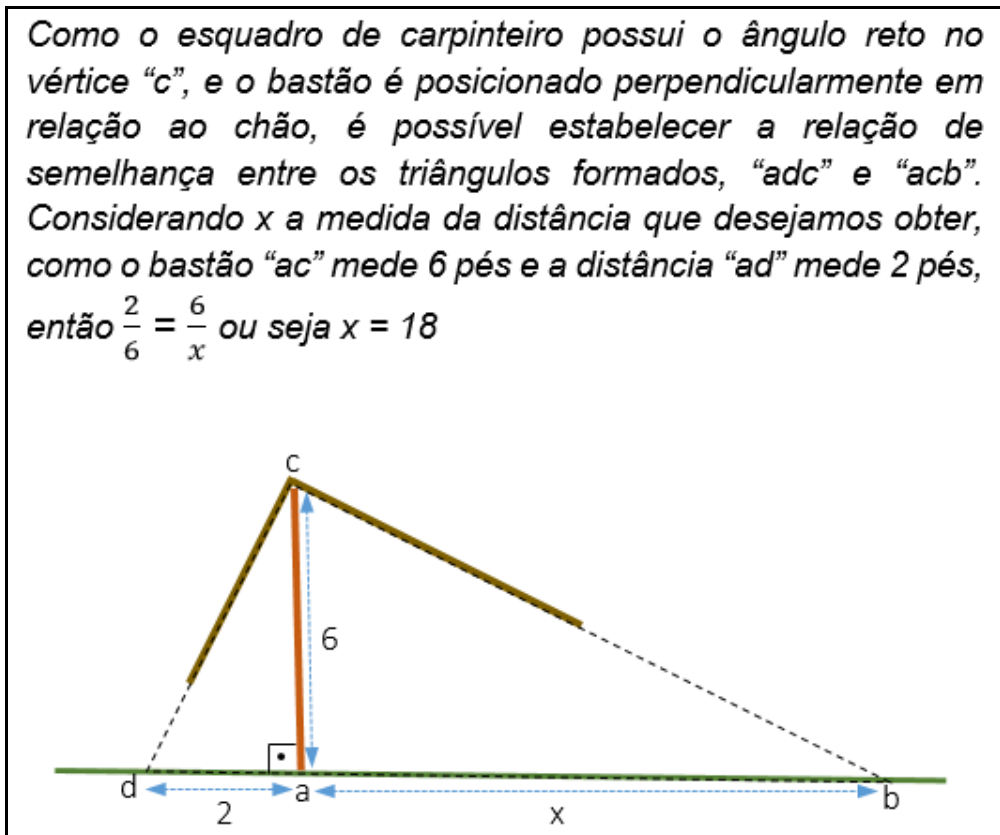

Figura 7 - Justificativa matemática - medição de distância com o esquadro de carpinteiro

Fonte: Produção nossa

Também nestas orientações Leonard segue a ideia da proporcionalidade em triângulos semelhantes, porém faz uma ressalva ao reconhecer que o instrumento não é adequado para medir grandes distâncias. Esta limitação também pode ser explorada em sala de aula com a seguinte questão. Por que com o instrumento essa medida não é possivel? O que seria necessário para obter a medida de grandes distâncias?

\section{Considerações Finais}

A exploração de instrumentos matemáticos permite o trabalho didático que não envolva somente o caráter instrumental isto é não só como ferramentas para confirmar uma medida ou se chegar a um resultado, empregando relações métricas em um processo de medição, mas também como construtores de conhecimento na época de sua fabricação o que 
constitui uma análise da dimensão epistemológica desse conhecimento, já que para uma produção de instrumento diversos conhecimentos eram mobilizados e constituíam o saber matemático de uma época. O que traz uma rica discussão em sala de aula com professores de matemática a respeito do uso da História da Matemática como gatilho de reflexão no processo histórico de construção de conhecimento e constitui uma análise do contexto da época e também de como a história é escrita e divulgada constituindo uma análise historiográfica do tratado.

\section{Referências}

AMARAL, Izabel. Quase tudo que você queria saber sobre tectônica, mas tinha vergonha de perguntar. Pós - Revista do Programa de PósGraduação em Arquitetura e Urbanismo da FAUUSP, n. 26, p. 148-167, 2009.

CASTILLO, Ana Rebeca Miranda. Um estudo sobre os conhecimentos matemáticos incorporados e mobilizados na construção e no uso do báculo (cross-staff) em A boke named Tectonicon de Leonard Digges. 2016. 121 f. Tese (Doutorado em Educação Matemática). Pontifícia Universidade Católica de São Paulo, São Paulo, 2016.

DIGGES, Leonard. A booke named Tectonicon. London: Felix Kyngston, 1605.

Disponivel em:

https://ia802707.us.archive.org/17/items/bookenamedtecton00digg/bo okenamedtecton00digg_bw.pdf. Acesso em: 21 abr. 2019

HARKNESS, Deborah. E. The Jewel House: Elizabethan London and the scientific revolution. London: Yale University Press, 2007. 
HIGTON, Hester. Does using an instrument make you mathematical? Mathematical practitioners of the $17^{\text {th }}$ century. Endeavour, v. 25, n. 1, p. 18-22, 2001.

MCRAE, Andrew. To know one's own: Estate surveying and the representation of the land in early modern England. The Huntington Library Quarterly, p. 333-357, 1993.

RICHESON, Allie Wilson. English Land Measuring to 1800: Instruments and Practices. Cambridge/London: The Society for the History of Technology/The MIT Press, 1966.

TAYLOR, Eva Germaine R. The Mathematical Practitioners of Tudor \& Stuart England. Cambridge: Institute of Navigation/Cambridge University Press, 1954.

VAN DEN HOVEN, Birgit. Work in ancient and medieval thought: ancient philosophers, medieval monks and theologians and their concept of work, occupations and technology. Amsterdam: J. C. Gieben, 1996.

Artigo recebido em: 2 out. 2019 Artigo aprovado em: 28 out. 2019 